\title{
Infant hearing screening at primary healthcare immunisation clinics in South Africa: The current status
}

\author{
L Petrocchi-Bartal, MA; K Khoza-Shangase, $\mathrm{PhD}$ \\ Department of Speech Pathology and Audiology, School of Human and Community Development, University of the Witwatersrand, \\ Johannesburg, South Africa
}

Corresponding author: K Khoza-Shangase (katijah.khoza@wits.ac.za)

\begin{abstract}
Background. In the developing world, it is critical that the acceptable principle that early hearing detection and intervention (EHDI) programmes be contextually useful, given the extensive level of difficulties faced by these regions. The Health Professions Council of South Africa (HPCSA) has recommended specific contexts in which to actualise EHDI application. One of these contexts relates to hearing screening at immunisation clinics within the first 6 weeks of life.

Objective. To explore the current status of the implementation of the HPCSA's 2007 guidelines for clinic-based hearing screening within the South African primary healthcare clinic (PHC) setting.

Methods. Within a qualitative research design, 30 PHC nurses representing 30 PHC clinics in the North West and Gauteng Provinces were interviewed using a structured questionnaire. Qualitative as well as thematic content analysis strategies were adopted in analysing data. Results. There is a lack of formal EHDI implementation at PHC clinics in both provinces. Lack of equipment, budgetary constraints and human resource challenges are the reasons for this. Regardless of the province's socioeconomic development based on the deprivation index, EHDI implementation at clinic level is not feasible unless the barriers are addressed.

Conclusion. There is a need to ensure that context-specific studies in EHDI are conducted. This will ensure that national position statements are sensitive to contextual challenges and that they allow for evidence-based practice. This is particularly relevant in developing countries where resource constraints dictate the success or failure of any well-intentioned programme. The HPCSA's 2007 position statement needs to be reviewed taking careful cognisance of feasibility study findings such as the current one. Findings have implications for nurses training, future studies, and policy formulation, as well as clinical plans for EHDI in developing contexts.
\end{abstract}

S Afr J Child Health 2016;10(2):139-143. DOI:10.7196/SAJCH.2016.v10i2.1114

The negative influence that impaired hearing has on a child's development may be pervasive, affecting areas such as cognition, ${ }^{[1]}$ language, educational, social and emotional competence, ${ }^{[2]}$ literacy development, ${ }^{[3]}$ as well as eventual vocational ${ }^{[4]}$ and thus financial outcomes. $^{[3-5]}$ Decreased social burden and improved quality of life depend on the ability of a hearing screening programme to identify hearing problems in infants at the earliest opportunity. ${ }^{[6]}$ Internationally, positing universal newborn hearing screening (UNHS) as a function of early hearing detection and intervention (EHDI) has been accepted as a measure of best practice pertaining to child healthcare ${ }^{[1]}$ and has also been highlighted as the favoured approach for private and public sector hearing healthcare. ${ }^{[7,8]}$ As they pertain to audiology, early intervention principles include a proposal for diagnosis of hearing impairment, with early intervention services effected by 6 months of age internationally, ${ }^{[9]}$ and in South Africa (SA) by a maximum of 8 months of age. ${ }^{[10]}$ Many developed countries ${ }^{[9]}$ and some developing countries ${ }^{[11]}$ have in fact promulgated hearing screening policy based on Joint Committee on Infant Hearing principles. ${ }^{[9]}$ Most developing world contexts have not formally recognised the advantages that UNHS presents and have consequently not legislated any hearing screening protocols. ${ }^{[12]}$ The reasons for the lack of EHDI in these contexts are uncertain and complex. One can deduce that additional burdens often endemic to areas such as subSaharan Africa, including poverty ${ }^{[4]}$ and the burden of life-threatening diseases such as HIV/AIDS and $\mathrm{TB},{ }^{[13]}$ are of influence. In these contexts, hearing impairment may be viewed as relatively less urgent, which renders EHDI implementation as low priority. ${ }^{[1]}$ The acceptable precept that EHDI programmes must be contextually relevant is even more critical in the developing world, where the challenges faced are considerable when compared with developed world environments. ${ }^{[14]}$ Within sub-Saharan Africa, only Nigeria and SA have documented evidence on aspects of EHDI as they apply within varying contexts in this part of the developing world. ${ }^{[15]}$ In these countries, the available literature reveals a fragmented approach to gathering evidence, showing a need for systematic and thorough research into all aspects of EHDI. To date, not enough published evidence emanating from SA studies on EHDI exists; although this lacunae in the literature is slowly receiving increased attention. Three of these investigations are comparatively recent research efforts, with previous publications issued during the period 2006 to 2010. Within the private sector, Swanepoel et al. ${ }^{[16]}$ explored newborn hearing screening in an SA private healthcare hospital, and Meyer et al. ${ }^{[17]}$ investigated early detection of infant hearing loss in the SA private healthcare sector. Scheepers et al. ${ }^{[18]}$ examined the reasons parents in the SA context refuse hearing screening and default on follow-up screening. Four studies investigated different aspects of hearing screening within the government sector. Specifically, Swanepoel et al. ${ }^{[14]}$ conducted a study on infant hearing screening (IHS) at immunisation clinics in SA, Theunissen and Swanepoel ${ }^{[19]}$ investigated public health sector EHDI services in SA, Kanji et al. ${ }^{[20]}$ conducted a retrospective review on hearing screening follow-up return rates in very-low-birth-weight infants, and Friderichs et al..$^{[21]}$ analysed the efficacy of a communitybased hearing screening programme utilising existing clinic staff in the Western Cape. Most recently, Khoza-Shangase and Harbinson ${ }^{[22]}$ reported on the evaluation of UNHS in SA primary care. This paucity in evidence reflects a lack of realised hearing screening in different settings of community practice. As such, either directly or by implication, these studies recommend extensions of the current 
research scope for further expansion of knowledge in the field of EHDI in the SA context so as to enable the promulgation of evidencebased hearing screening protocols.

With appropriate combining of logistical and theoretical paradigms, key issues within the specific screening milieu must be identified and explored if inroads are to be made towards realising the ultimate aim of decreasing disability through application of a contextually relevant UNHS programme. The current study explored the feasibility of implementing the Health Professions Council of South Africa (HPCSA) 2007 guidelines for clinic based hearing screening in the SA primary healthcare clinic (PHC) setting.

\section{Objective}

The main objective of the study was to explore the feasibility of implementing the HPCSA's 2007 guidelines for clinic-based hearing screening in the SA PHC setting. Specific objectives included establishing trends of the number of IHS programmes conducted at PHC immunisation clinics in the North West Province (NWP) and Gauteng Province (GP), as well as determining and exploring the possible factors considered to influence the implementation of IHS programmes at this level of healthcare.

\section{Methods}

Ethical clearance was secured from the University of the Witwatersrand's Human Ethics Committee (Medical) (protocol no.: M091040). Through non-probability purposive sampling, 30 PHC, English-speaking clinic nurses in charge of $30 \mathrm{PHC}$ immunisation clinics within the NWP and GP (15 per province) were recruited according to accessibility and according to the district demographic classification. ${ }^{[23]}$ These nurses were either officially within the position of operations manager or acting operations manager, or heading the clinic at the time if the incumbent was unavailable for the interview. The main reason the clinic manager was selected as interviewee was that being at the helm implies insight as to the detailed workings of the particular clinic in question. It is within these clinics where yield (actual immunisations generated out of the possible population in the area) is reported to be $91.8 \%$ in GP and $71.4 \%$ in the NWP. ${ }^{[23]}$ Community nurses and lay volunteers within the maternal child woman's health cluster provide services, including implementation of immunisation programmes. These clinics incorporate the specific targeted population of infants requiring immunisations at 6,10 and 14 weeks, 9 and 18 months, and 5 years, and may be described as predominantly outpatient facilities. ${ }^{[24]}$

As estimated from the Community Survey's deprivation index, ${ }^{[25]}$ the NWP population group is considered to be more disadvantaged socioeconomically than that of $\mathrm{GP}^{[23]}$ It is because of these demographic differences that the two provinces were selected, where these overall provincial differences were aimed at facilitating a ruralurban divide. These were considered to be useful for determining hearing healthcare outcome determinants within the context of the current study. Specific districts within these provinces were selected.

The research design adopted was qualitative in nature, with a questionnaire administered in a structured face-to-face interview with each participant. The interview location (for all interviews including those conducted for the pilot project) was the participant's immunisation clinic. The interview schedule and questionnaire used were structured, where most questions presented were factual and closed ended. These were supplemented by several open-ended questions to enable documentation of free thought processes that the researcher transcribed verbatim. The questionnaire was adapted from a self-administered questionnaire previously used in a study conducted in a collaborative effort by EHDI SA, the Centre for Deaf Studies and Deaf Education, the University of Pretoria and the University of the Witwatersrand. ${ }^{[19]}$ The structured questionnaire consisted of demographic information, information related to work context and hearing screening context, as well as information management and quality control measures. The interviews were also tape recorded. Data were collected via verbatim documentation of respondents' answers and audio-taped recordings of interviews, which were transcribed. The researcher asked questions in numerical order to ensure consistency of format and organisation between interviews. Question explanation and probing was not applied beyond the methodological protocol in order to ensure consistency between interviews. Conducting consistent and structured interview sessions, as per the procedures delineated, ensured uniformity between and within interviews in order to enhance the study's reliability. Subject bias, such as the Hawthorne effect where participants have a heightened awareness that they form part of an evaluation process, may have influenced performance in the current study; however, the researcher attempted to minimise the impact of this by having verbally ensured participant confidentiality with regard to their responses, with the added assurance that their job security could not be affected in any way. The time frame for administering the structured interviews spanned a maximum of 1 hour per interview. Data were analysed qualitatively and through thematic content analysis, where transcriptions were evaluated to determine and code the emerging themes. Quantitative data analysis, through the use of frequency calculations, was condensed into tabular format for ease of frequency comparison between regions. Comparisons and contrasts were evaluated between the two provincial sample groups and their sub-groups located in the NWP and GP.

In order to ensure research reliability, controls were exercised pertaining to participant variables as well as with regard to those relating to the questionnaire used to obtain data. Careful control of the consistency of the interview procedures employed was also ensured. To further ensure reliability and validity, site observations as well as use of an independent rater during data analysis were done over and above a pilot study on three nurses who did not form part of the main study to pretest the data collection tool and procedures prior to the main study. The first author was the sole interviewer of the nurses; she visited the sites and was able to view and describe the facilities.

\section{Results}

Data from the current study allowed for a clear depiction of the current status of newborn IHS programmes conducted at PHC immunisation clinics in the NWP and GP. Furthermore, useful and practical rationale influencing implementation of such services was obtained. Thematic content analysis as well as qualitative descriptive data analysis allowed for themes to be drawn from the data, with frequencies for such themes (Tables 1 and 2).

As depicted in Table 1, none of the PHC clinics offer or provide formalised newborn IHS. All participants reported that their clinics did not have the equipment to provide hearing screening. Of further interest is that $40 \%$ of all respondents (8 GP and 4 NWP respondents) felt that general budgetary issues were plausible reasons for the absence of hearing screening services at their clinics, with an overwhelming majority of respondents $(86.7 \%)$ regarding human resource (HR) constraints to be the major contributing factor to the lack of hearing screening services offered. Although only cited by $10 \%$ of respondents ( 3 NWP respondents), district issues (referred to as management decisions made at a district level) were considered to be an influence in rendering newborn IHS services. NWP respondents elaborated that if the matter was not sanctioned at a district level, provision of a hearing screening service would not be taken seriously and would not be implemented.

When comparing the number of newborn IHS between the two provinces, findings reflected that all GP respondents related the lack of newborn/IHS service delivery to HR issues, with $53.3 \%$ of GP respondents attributing lack of hearing screening services 
Table 1. Number of newborn/IHS programmes conducted/not conducted in each province and rationale $(N=30)$

\begin{tabular}{|c|c|c|c|}
\hline Issue & GP $(n=15), n(\%)$ & NWP $(n=15), n(\%)$ & Total $(N=30), n(\%)$ \\
\hline $\begin{array}{l}\text { Is formalised hearing } \\
\text { screening conducted? }\end{array}$ & No, $15(100)$ & No, $15(100)$ & No, $30(100)$ \\
\hline $\begin{array}{l}\text { Is formalised hearing } \\
\text { screening equipment } \\
\text { available? }\end{array}$ & No, $15(100)$ & No, $15(100)$ & No, $30(100)$ \\
\hline $\begin{array}{l}\text { Is budgetary } \\
\text { allocation the reason } \\
\text { for the above? }\end{array}$ & $\begin{array}{l}\text { Yes, } 8(53.3) \\
\text { No, } 0(0) \\
\text { Maybe, } 7(46.7)\end{array}$ & $\begin{array}{l}\text { Yes, } 4(26.7) \\
\text { No, } 1(6.7) \\
\text { Maybe, } 7 \text { (46.7) } \\
\text { District level issue, } \\
3(20)\end{array}$ & $\begin{array}{l}\text { Yes, } 12(40) \\
\text { No, } 1(3.3) \\
\text { Maybe, } 14 \text { (46.7) } \\
\text { District level issue, } 3 \text { (10) }\end{array}$ \\
\hline $\begin{array}{l}\text { Is HR allocation the } \\
\text { reason for the lack of } \\
\text { formalised hearing } \\
\text { screening? }\end{array}$ & Yes, 15 (100) & $\begin{array}{l}\text { Yes, } 11(73.3) \\
\text { No, } 3(20) \\
\text { Maybe, } 1(6.7)\end{array}$ & $\begin{array}{l}\text { Yes, } 26(86.7) \\
\text { No, } 3(10) \\
\text { Maybe, } 1(3.3)\end{array}$ \\
\hline $\begin{array}{l}\text { Are HPCSA hearing } \\
\text { screening guidelines } \\
\text { followed? }\end{array}$ & No, $15(100)$ & No, $15(100)$ & No, $30(100)$ \\
\hline \multirow{8}{*}{$\begin{array}{l}\text { What equipment } \\
\text { is used for hearing } \\
\text { screening? (as per } \\
\text { themes raised) }\end{array}$} & \multicolumn{3}{|c|}{ Otoscopic examination sometimes used } \\
\hline & $\begin{array}{l}\text { Yes, } 14(93.3) \\
\text { No, } 1(6.7)\end{array}$ & $\begin{array}{l}\text { Yes, } 14(93.3) \\
\text { No, } 1(6.7)\end{array}$ & $\begin{array}{l}\text { Yes, } 28(93.3) \\
\text { No, } 2(6.7)\end{array}$ \\
\hline & \multicolumn{3}{|c|}{$\begin{array}{l}\text { At Road to Health Chart immunisations or milestone review, baby's } \\
\text { behavioural responses to environmental sound stimuli incorporating } \\
\text { noisemaker and/or verbal stimuli assessed }\end{array}$} \\
\hline & Yes, $6(40)$ & Yes, $3(20)$ & Yes, $9(30)$ \\
\hline & \multicolumn{3}{|c|}{ Reviewing of medical records when an auditory problem was indicated } \\
\hline & Yes, $10(67)$ & Yes, $13(86.7)$ & Yes, $23(76.7)$ \\
\hline & \multicolumn{3}{|c|}{$\begin{array}{l}\text { Reviewing of medical records at Road to Health Chart immunisations } \\
\text { or milestone review }\end{array}$} \\
\hline & Yes, $8(53.3)$ & Yes, $11(73.3)$ & Yes, 19 (63.3.3) \\
\hline $\begin{array}{l}\text { Are HPCSA hearing } \\
\text { screening guidelines } \\
\text { used? }\end{array}$ & No, $15(100)$ & No, $15(100)$ & No, $30(100)$ \\
\hline
\end{tabular}

Table 2. Number of newborn/his programmes and rationale - HR reasons provided $(N=30)^{*}$

\begin{tabular}{|c|c|c|c|}
\hline HR issue/theme raised & GP $(n=15), n(\%)$ & NWP $(n=15), n(\%)$ & Total $(N=30), n(\%)$ \\
\hline HR budgetary issue? & Theme not raised & Yes, 1 (6.7) & Yes, 1 (3.3) \\
\hline Reduced staff training & $10(66.7)$ & $3(20)$ & $13(43.3)$ \\
\hline $\begin{array}{l}\text { Unspecified general } \\
\text { budgetary issue }\end{array}$ & $1(6.7)$ & $2(13.3)$ & $3(10)$ \\
\hline Staff shortage & $2(13.3)$ & $2(13.3)$ & $4(13.3)$ \\
\hline Space & Theme not raised & $1(6.7)$ & $1(3.3)$ \\
\hline Unsure & $1(6.7)$ & Theme not raised & $1(3.3)$ \\
\hline
\end{tabular}

rendered to general budgetary constraints (referred to as general lack of government funding to PHC service providers). In contrast, $73.3 \%$ of NWP respondents related the lack of newborn IHS service delivery to $\mathrm{HR}$ issues, with $26.7 \%$ citing general budgetary issues to the lack of such services rendered. Similar trends were present at a sub-district level. Table 2 shows results from further in-depth analysis of HR issues. The total sample $(N=30), 43.3 \%$ of respondents (mainly from GP) considered the lack of appropriate training in newborn or IHS to be a central reason that influenced the provision of newborn/IHS services. This was followed by the staff shortages as mentioned by $13.3 \%$ of respondents. NWP highlighted a spread of HR related factors such as (but not limited to) budgetary issues and staff shortages.

\section{Discussion}

Current findings revealed that no PHC clinics within the NWP and GP were conducting formalised hearing screening as promulgated by the HPCSA 2007 EHDI position statement. Budgetary and HR issues (mainly staff training and staff shortages) were highlighted as the main reasons for this lack of formalised hearing screening implementation. It appears that economic indicators may have impinged on logistical and operational factors such as the clinic's physical structure, staff training, equipment availability and staff complement. The reported inequities in district finance distribution ${ }^{[26]}$ may have contributed to the differences in responses obtained between provinces.

Current findings reveal a rather depressing picture about the feasibility of the implementation of the HPCSA 2007 position statement on EHDI at PHC clinics; a level of healthcare most accessible to the majority of the SA population. Inability and failure to implement EHDI has significant implications that have been well documented, although these are often not seen as an important health priority when the burden of disease is as high as it is in SA. Nonetheless, current findings are viewed in light of the WHO's definition of health. The WHO defines health to not only encompass the absence of infirmity or disease, but to include the individual's complete physical, mental and social wellbeing. ${ }^{[27]}$ This reflects a tenet beyond the concept of survival and emphasises the urgent need for EHDI to improve life outcomes beyond existence for those infants with hearing loss. Early hearing intervention services, inclusive of early identification, are considered the foundation for attaining most favourable results in infants with hearing loss. ${ }^{[28]}$ Unfortunately, the generally less resourced public healthcare sector within SA's developing world context, is the option upon which $85 \%$ of the population relies. ${ }^{[28]}$ If current findings are replicated throughout SA's nine provinces; a less than favourable picture is drawn for the public sector. Arguably one might rationalise these findings when considering the burden of disease. Globally, SA has one of the highest burden of $\mathrm{TB}$ and HIV/AIDS. ${ }^{[29]}$ These diseases continue to receive precedance over non life- threatening conditions such as hearing loss. ${ }^{[1]}$ EHDI services within the SA context, are in their infancy with very little 
contextually relevant evidence for their efficacy and applicability. ${ }^{[30]} \mathrm{A}$ paucity of scientific data as well as a lack of investigative studies into EHDI implementation in the SA context prevails. ${ }^{[16,18]}$ Current findings highlight and provide evidence that supports the importance of advocating for the responsibility of legislation mandating EHDI, which is currently lacking. ${ }^{[28]}$ Current evidence indicates that primary alerts to possible hearing loss lie with the caregiver. Here their concerns may relate to speech-language delays, atypical behaviour or otitis media complications. ${ }^{[15]}$ Hearing screening is not occurring at PHC clinics and this is where most mothers or caregivers take their infants and neonates for healthcare.

As per the HPCSA recommendations, the current study aimed to provide supportive contextual evidence as input to concretely guide the SA EHDI actualisation process. Fundamental to the current study was specific consideration to theoretical constructs and clinical outcomes in the SA PHC immunisation clinic context. Based on the current findings, it can be strongly argued that implementing the HPCSA's EHDI clinic guidelines does not appear feasible at this stage because of the overriding barriers revealed, although assets within the PHC immunisation context were identified. These central barriers, which included reduced clinic infrastructure and resources and reduced staff complement and training, preclude successful EHDI implementation. An overwhelming $86.7 \%$ of the participants reported HR constraints, $40 \%$ presented budget challenges, $43.3 \%$ believed lack of appropriate training in EHDI the significant barriers which should be taken seriously. A range of assets were identified and these included overall respondent willingness to implement formalised hearing screening as part of the PHC immunisation schedule. Other assets included patient return rates for immunisation, highlighting the favourable context the immunisation programme offers in terms of high patient yield. This is regarded as fundamental in a UHS programme. ${ }^{[15]}$

The unexpected differences between provinces and districts within provinces, with specific mention by NWP of the important role of district level governance may be related to differences within the specific regional and district management styles and priorities, especially given the greater level of authority the individual district is now able to exercise. ${ }^{[26]}$ Within the Department of Health (DoH) strategic plan, decentralised management of health districts has been strengthened for improved local accountability. ${ }^{[26]}$ The HPCSA 2007 position statement recommends inclusion of the responsible $\mathrm{DoH}$ agency to jointly facilitate the appropriate hearing screening programme. ${ }^{[10]}$ In this instance, it would appear that district level direct involvement in recommended hearing screening protocols is paramount in terms of the hearing screening programme's fit and application, where newborn/IHS may need to be more flexibile than implied by HPCSA 2007 recommended protocols. In addition, not only has inequity in DoH provincial financing been described, but inequality in DoH district financing has also been reported. ${ }^{[25]}$ Swanepoel et al. ${ }^{[28]}$ acknowledge that the SA public health sector is often less resourced than the state-of-the-art private health care sector. Theunissen and Swanepoel ${ }^{[19]}$ refer to a lack of equipment and staff shortages as the major contributing factors to reduced NHS services in the SA context, evidence that is consistent with current findings. What was unexpected was the extent to which the lack of staff training was cited as the main contributing reason for the lack of newborn/IHS service provision at a PHC level.

It is therefore recommended that district level DoH financing, participation and autonomy be considered when negotiating inclusion of newborn/IHS services with varied government level departments. It is suggested that specific $\mathrm{DoH}$ district policies and protocols be investigated in depth to ascertain specific levels of district financing and autonomy and how these relate to hearing healthcare service delivery.
Although current findings have significance in the EHDI initiatives in SA, they should be interpreted with due consideration of study design and analysis limitations identified. Firstly; the sample size of 30 clinics in only two provinces limits the generalisability of the findings. and this is raised as an implication for future studies. Secondly, this study only focused at one level of care, that of PHC. One can argue, although without evidence, that hearing screening occurs at other levels of healthcare such as secondary and tertiary levels. This is an implication for future studies.

\section{Conclusion}

Current findings at $\mathrm{PHC} /$ clinic level highlight the importance of carefully constructed studies investigating the feasibility of HPCSA 2007 protocol implementation. Continuously assessing feasibility of guidelines and/or position statements not only ensures evidencebased practice; but also enforces programme implementation that is contextually relevant and specific at any given point in time. This is particularly true where programme implementation can be significantly influenced by barriers such as resource allocations.

Acknowledgements. A faculty ad hoc grant and a faculty research promotion grant from the University of the Witwatersrand were received and enabled this research to be conducted.

\section{References}

1. Olusanya BO. Can the world's infants with hearing loss wait? Int J Pediatr Otorhinolaryngol 2005;69:735-738. DOI:10.1016/j.ijporl.2005.03.024

2. Northern JL, Downs MP. Hearing in Children. 4th ed. Baltimore: Williams \& Wilkins; 1991:28-31.

3. DesJardin JL, Ambrose SE, Martinez AS, Eisenberg LS. Relationships between speech perception abilities and spoken language skills in young children with hearing loss. Int J Audiol 2009;48(5):248-259. DOI:10.1080/149920208 02607423

4. Olusanya BO. Hearing impairment prevention in developing countries: making things happen. Int J Pediatr Otorhinolaryngol 2000;55(3):167-171. DOI:10.1016/S0165-5876(00)00392-X

5. Olusanya BO, Ruben R, Parving A. Reducing the burden of communication disorders in the developing world: An opportunity for the millenium development project. J Am Med Assoc 2006;296:441-444. DOI:10.1001/ jama.296.4.441

6. Hutt N, Rhodes C. Post-natal hearing loss in universal neonatal hearing screening communities: Current limitations and future directions. J Pediatr Child Health 2008;44(3):87-91. DOI:10.111/j.1440-1754.2007.01275.x

7. Joint Committee on Infant Hearing. Year 2000 position statement: Principles and guidelines for early hearing detection and intervention programs. Pediatrics 2000;106(4):798-817. DOI:10.1044/1059-0889(2000/005)

8. Olusanya BO, Luxon LM, Wirz SL. Screening for early childhood hearing loss in Nigeria. J Med Screen 2005;12(3):115-118. DOI:10.1258/0969141054855274

9. Joint Committee on Infant Hearing Screening. Year 2007 position statement Principles and guidelines for early hearing detection and intervention programs. Pediatrics 2007;120(4):898-92. DOI:10.1542/peds.2007-2333

10. Health Professions Council of South Africa. Early Hearing Detection and Intervention Programmes in South Africa, Position Statement Year 2007. http://www.hpcsa.co.za/hpcsa/UserFiles/Files/Speech\%20language\%20and\% 20hearing/EHDI5position\%20statement\%20(HPCSA\%2007).pdf (accessed 29 October, 2010)

11. Yee-Arellano HM, Leal-Garza F, Pauli-Muller K. Universal newborn hearing screening in Mexico: Results of the first 2 years. Int J Pediatr Otorhinolaryngo 2006;70:1863-1870

12. Olusanya BO. Addressing the global neglect of childhood hearing impairment in developing countries. PLoS Med 2007;4(4):626-630. DOI:10.1371/journal. pmed.0040074

13. Streefland P. Public health care under pressure in sub-Saharan Africa. Health Policy 2005;71(3):375-382. DOI:10.1016/j.healthpol.2004.06.002

14. Swanepoel de W, Louw B, Hugo R. A novel service delivery model for infant hearing screening in developing countries. Int J Audiol 2007;46(6):321-327. DOI:10.1080/14992020601188583

15. Olusanya BO, Okolo AA. Early hearing detection at immunization clinics in developing countries. Int J Pediatr Otorhinolaryngol 2006;70(8):1495-1498. DOI:10.1016/j.ijporl.2006.04.002

16. Swanepoel D, Ebrahim S, Joseph A, Friedland PL. Newborn hearing screening in a South African private health care hospital. Int J Pediatr Otorhinolaryngo 2007;71(6):881-887. DOI:10.1016/j.ijporl.2007.02.009

17. Meyer ME, Swanepoel DW, Le Roux TL, Van der Linde M. Early detection of infant hearing loss in the private health care sector of South Africa. Int Otorhinolaryngol 2012;76(5):698-703. DOI:10.1016/j.ijporl.2012.02.023 
18. Scheepers LJ, Swanepoel DW, Le Roux TL. Why parents refuse newborn hearing screening and default on follow-up rescreening - A South African perspective. Int J Pediatr Otorhinolaryngol 2014;78(4):652-658. DOI:10.1016/ j.jporl.2014.01.026

19. Theunissen M, Swanepoel DW. Early hearing detection and intervention services in the public health sector in South Africa. Int J Audiol 2008;47(Supplement1):S23-S29. DOI:10.1080/14992020802294032

20. Kanji A, Khoza-Shangase K, Ballot D. Hearing screening follow-up return rate in a very low birth weight project: A retrospective record review. S Afr J Child Health 2010;4(4):95-99.

21. Friderichs N, Swanepoel DW, Hall J. Efficacy of a community-based hearing screening program utilizing existing clinic personnel in Western Cape, South Africa. Int J Pediatr Otorhinolaryngol 2012;76(4):552-559. DOI:10.1016/j. ijporl.2012.01.015

22. Khoza-Shangase K, Harbinson S. Evaluation of universal newborn hearing screening in South African primary care, African Journal of Primary Health Care \& Family Medicine 2015;7(1):12. DOI:10.4102/phcfm.v7i1.769

23. Day C, Gray A. Indicators: Health and Related Indicators. South Africa Health Review 2008. Durban: The Health Systems Trust, 2008.

24. Office of the President, K Motlanthe. SA yearbook 2008/2009, health chapter
12. In: Burger D, ed. SA Yearbook 2008/2009, 16th ed. Pretoria: Government Communication and Information System (GCIS), 2009:310-331.

25. Statistics South Africa. Community Survey 2007 (revised version), (Statistical Release P30301). Pretoria: Statistics South Africa, 2007. http://www.statssa.gov. $\mathrm{za} /$ PublicationsHTML/P03112997/html/P030112007.html (accessed 16 June 2010).

26. National Department of Health, South Africa. Strategic plan 2010/11 2012/13. Pretoria: NDoH, 2010. http://www.nationalplanningcycles.org/sites/ default/files/country_docs/South\%20Africa/south_africa_strategic_health_ plan 2010-2013.pdf (accessed 29 May 2010).

27. World Health Organization. WHO definition of health, 2003. http://who.int/ about/definition/en/print.html (accessed 1 July 2010).

28. Swanepoel D, Storbeck C, Friedland P. Early hearing detection and intervention in South Africa. Int J Pediatr Otorhinolaryngol 2009;73(6):783-786. DOI:10.1016/j.ijporl.2009.01.007

29. Matjila MJ, Hoosen AA, Stolz A, Cameron N. STIs, HIV and TB: Progress and challenges. In: Barron P, Roma-Reardon J, eds. South African Health Review. Durban: Health Systems Trust, 2008.

30. Swanepoel DC, Delport SD, Swart GJ. Universal newborn hearing screening in South Africa - a first-world dream? S Afr Med J 2004;94(8):634-635. 